\title{
Guías para el tratamiento de la dislipidemia en pacientes con VIH/SIDA que reciben TARGA
}

\author{
Benhard Hasbum, Antonio Solano, María Paz León, Ricardo Boza.
}

\section{Resumen}

La dislipidemia es una de las complicaciones más frecuentes en los pacientes infectados por el VIH que reciben tratamiento antiretroviral. Su manejo se ve complicado por las interacciones entre algunos de las terapias hipolipemiantes y los inhibidores de proteasa. Estas guías buscan facilitar el abordaje de estos casos tanto en el diagnóstico, su clasificación y manejo no farmacológico como farmacológico.

Descriptores: VIH, dislipidemia, estatinas, fibratos, riesgo cardiovascular.

Key words: HIV, dyslipidemia, statins, fibrates, cardiovascular risk

Recibido: 3 de mayo de 2005

Aceptado: 31 de mayo de 2005

\section{Antecedentes}

El advenimiento de la terapia antirretroviral de gran actividad (TARGA) a modificado sustancialmente el perfil de morbi-mortalidad de los pacientes infectados por el virus de inmunodeficiencia humana (VIH). Antes del empleo sistemático de esta terapia las alteraciones metabólicas que con mayor frecuencia se describían en estos pacientes estaban en relación con la diarrea crónica, la desnutrición y el síndrome de desgaste ${ }^{1}$.

La aparición de eventos vasculares aterotrombóticos en pacientes jóvenes que recibían la TARGA, en especial aquellos esquemas que incluían un inhibidor de proteasas, hizo sospechar por primera vez en 1998 que esta terapia podría estar relacionada con la inducción de un perfil metabólico adverso para el endotelio vascular ${ }^{2}$.

Aunque no existe todavía evidencia concreta que demuestre que la enfermedad cardiovascular es más prevalente en los pacientes con VIH/SIDA que reciben TARGA ${ }^{3}$, se ha relacionado a esta terapia con la aparición de lipodistrofia, insulino-resistencia, disminución del colesterol HDL, aumento del colesterol LDL y VLDL, así como elevación de los niveles plasmáticos de triglicéridos ${ }^{4}$.

Según diversos reportes la prevalencia de dislipidemia en pacientes que reciben TARGA

Abreviaturas: TARGA, terapia antirretroviral de gran cantidad; VIH, virus inmunodeficiencia humana; NCEPIII tercer panel de expertos en colesterol; IP, inhibidores de proteasa.

ISSN 0001-6002/2005/47/3/144-147 Acta Médica Costarricense, (C2005 Colegio de Médicos y Cirujanos alcanza el $60 \%$, siendo en tres cuartas partes de los casos del tipo Fredrickson IV (VLDL alto con LDL normal) ${ }^{5}$. No se conocen con certeza los mecanismos moleculares implicados en estas alteraciones, aunque se ha relacionado más a los IP y a los inhibidores de la transcriptasa inversa análogos nucleósidos que a los inhibidores de la transcriptasa inversa no análogos nucleósidos ${ }^{4}$. Incluso dentro de cada grupo, se han demostrado diferencias sustanciales entre los diversos fármacos.

La estavudina al ser comparada con el tenofovir produjo una elevación temprana y estadísticamente significativa en los niveles de triglicéridos y colesterol total ${ }^{6}$. Dentro de los IP el ritonavir, cuando de utiliza a dosis de antiretroviral, ha demostrado las elevaciones más 
marcadas en los niveles plasmáticos de triglicéridos. El atazanavir, IP aprobado recientemente por la Food and Drug Administration de los EEUU, no se reporta que altere el perfil lipídico. El fosamprenavir y el saquinavir tienen un efecto mínimo. La combinación lopinavir/ritonavir no se describe que altere los niveles de HDL, pero afecta negativamente los de triglicéridos y colesterol total ${ }^{7}$.

Los antecedentes familiares de dislipidemia, los hábitos nutricionales y los niveles de colesterol y triglicéridos antes de iniciar la TARGA se han relacionado con la aparición posterior de dislipidemia ${ }^{1}$.

\section{Abordaje}

El tratamiento de la dislipidemia en el paciente con VIH/SIDA es aún objeto de mucha controversia. Existe una carencia importante de estudios prospectivos, placebo controlados que demuestren los efectos del tratamiento hipolipemiante en pacientes con VIH/SIDA. No se ha demostrado que el tratamiento crónico con estatinas, fibratos o una combinación de ambos prevenga eventos cardiovasculares en estos pacientes. Además debe tenerse en cuenta que la TARGA se relaciona a múltiples interacciones farmacológicas, que pueden inactivar su función o aumentar su toxicidad ${ }^{8}$.

En la evaluación inicial de todo paciente con dislipidemia debe llevarse a cabo una historia clínica completa. Hay que explorar la existencia de antecedentes familiares de dislipidemia que sugieran la presencia de un transtorno genético subyacente (hipercolesterolemia familiar o hiperlipemia familiar combinada) así como la presencia de enfermedades subyacentes (ej. Diabetes mellitus tipo-2, hipotiroidismo) y tratamientos farmacológicos (ej. esteroides, retinoides) con potencial deletéreo sobre el perfil lipídico. La presencia, en la evaluación clínica, de arco senil, lipemia retinalis, xantomas o xantelasmas sugiere la existencia de un transtorno genético ${ }^{\text {. }}$

No existe una guía específica para la evaluación inicial de la dislipidemia en los pacientes con VIH/SIDA que reciben TARGA. Tampoco existe evidencia de que el estado inflamatorio crónico que se observa en estos pacientes promocione la formación de placas ateromatosas a niveles de colesterol inferiores.

De esta manera, para el abordaje y seguimiento de los pacientes con VIH/SIDA que reciben TARGA y presentan elevaciones séricas de colesterol se recomienda la aplicación de las guías del Tercer Panel de Expertos en Colesterol (NCEP III) donde se clasifica a los pacientes según los factores de riesgo cardiovascular, los niveles de colesterol LDL y las diferentes consideraciones terapéuticas (ver cuadro ) ${ }^{10}$.

Se consideran factores de riesgo cardiovascular: hombre $>45$ años, mujer $>55$ años, colesterol HDL $<40 \mathrm{mg} / \mathrm{dl}$, presión arterial $>140 \mathrm{mg} / \mathrm{dl}$ o uso de tratamiento antihipertensivo, tabaquismo e historia familiar de enfermedad coronaria en familiares de primer grado en hombres $<55$ años y mujeres $<65$ años ${ }^{9}$.

En lo referente a los triglicéridos, se toman en consideración los siguientes parámetros: elevación limítrofe: $150-199 \mathrm{mg} / \mathrm{dl}$, niveles elevados : $200-499 \mathrm{mg} / \mathrm{dl}$ y niveles muy elevado : $>500 \mathrm{mg} / \mathrm{dl}$. Cuando existen niveles elevados se indica tratamiento con estatinas si el colesterol no HDL (colesterol total - HDL) supera en $30 \mathrm{mg} / \mathrm{dl}$ el nivel límite de LDL para ese paciente. Para los niveles muy elevados se sugiere el empleo de fibratos ${ }^{\text {? }}$.

\begin{tabular}{|c|c|c|c|}
\hline \multicolumn{4}{|c|}{ Cuadro 1. Riesgo cardiovascular establecido por el NCEP III } \\
\hline Categoría & Meta LDL-C & $\begin{array}{l}\text { Intervención no } \\
\text { farmacológica }\end{array}$ & Fármacos \\
\hline $\begin{array}{c}\text { Alto riesgo : Enf. Cardiovascular o } \\
\text { equivalente } \dagger \\
\text { Riesgo a } 10 \text { años }>20 \%\end{array}$ & $\begin{array}{l}<100 \mathrm{mg} / \mathrm{dl} \\
\text { Meta opcional : } \\
\quad<70 \mathrm{mg} / \mathrm{dl}\end{array}$ & $\geq 100 \mathrm{mg} / \mathrm{dl}$ & $\geq 100 \mathrm{mg} / \mathrm{dl}$ \\
\hline $\begin{array}{l}\text { Riesgo moderadamente alto: } \\
2 \text { o más factores de riesgo. } \\
\text { Riesgo a } 10 \text { años } \\
10 \text { a } 20 \%\end{array}$ & $<130 \mathrm{mg} / \mathrm{dl}$ & $\geq 130 \mathrm{mg} / \mathrm{dl}$ & $\begin{array}{c}\geq 130 \mathrm{mg} / \mathrm{dl} \\
100-129 \mathrm{mg} / \mathrm{dl} \\
\text { considerar tratamiento } \\
\text { farmacologico }\end{array}$ \\
\hline $\begin{array}{c}\text { Riesgo moderado } \\
2 \text { o más factores de riesgo. } \\
\text { Riesgo a } 10 \text { años }<10 \%\end{array}$ & $<130 \mathrm{mg} / \mathrm{dl}$ & $\geq 130 \mathrm{mg} / \mathrm{dl}$ & $\geq 160 \mathrm{mg} / \mathrm{dl}$ \\
\hline $\begin{array}{l}\text { Bajo riesgo : < } 2 \\
\text { factores de riesgo }\end{array}$ & $<160 \mathrm{mg} / \mathrm{dl}$ & $\geq 160 \mathrm{mg} / \mathrm{dl}$ & $\begin{array}{l}\geq 190 \mathrm{mg} / \mathrm{dl} \\
160-189 \mathrm{mg} / \mathrm{dl}: \\
\text { tratamiento opcional }\end{array}$ \\
\hline $\begin{array}{l}\text { enf. arterial periférica, aneurisma } \\
\text { ente : Circulation } 2004 ; 110: 227-39\end{array}$ & arterial carotí & betes & \\
\hline
\end{tabular}




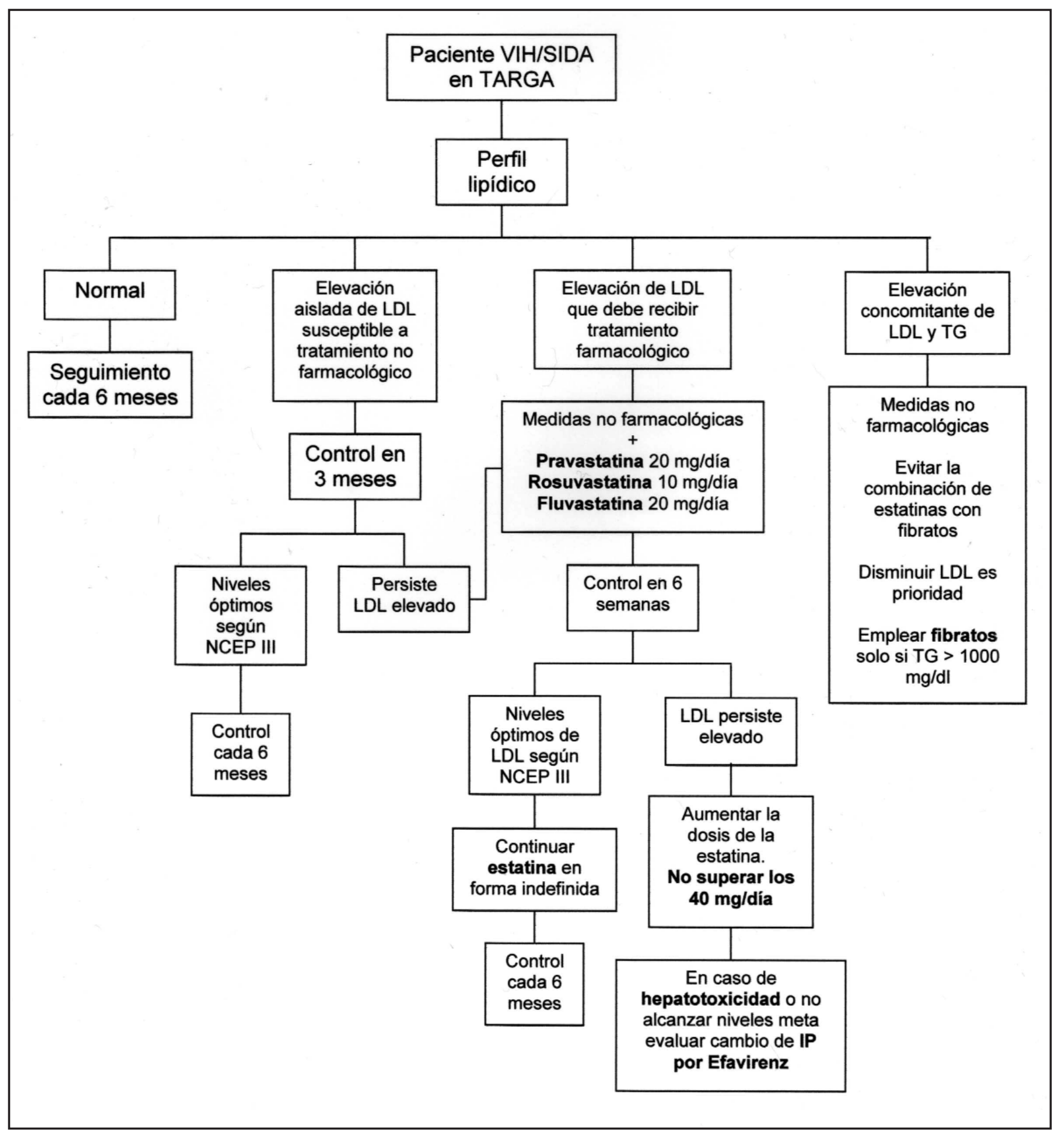

Figura 1. Algoritmo de manejo del paciente VIH con dislipidemia. 


\section{Tratamiento no farmacológico}

- Implementación de un programa de ejercicio aeróbico regular

- Suspensión del hábito tabáquico

- Moderar el consumo de bebidas alcohólicas

- Evaluar suspensión de fármacos hiperlipemiantes

- Dieta : carbohidratos : 50 a $60 \%$ del total de calorías diarias ( tcd ), proteínas : $15 \%$ tcd, grasa : $25-35 \%$ tcd ( grasa saturada : $7 \%$ tcd, grasa polinsaturada : $10 \%$ tcd, grasa monoinsaturada : $20 \%$ tcd ), $<200 \mathrm{mg} /$ día de colesterol y 20-30 gramos de fibra por día ${ }^{\text {. }}$

- Control de enfermedades crónicas concomitantes : diabetes, hipertensión, obesidad, síndrome de ovario poliquístico, hipotiroidismo

- La respuesta al tratamiento no farmacológico se evaluará a los 3 meses de su implementación

\section{Tratamiento farmacólogico (Figura)}

- Todo paciente que reciba TARGA debe tener un control periódico ( al menos cada 6 meses ) de los parámetros lipídicos

- Existe todavía poca información acerca del uso de las estatinas y los fibratos en el tratamiento de la dislipidemia de los pacientes VIH/SIDA que reciben TARGA

- Existen numerosas interacciones farmacológicas reportadas en los pacientes que reciben TARGA, especialmente por inhibición o inducción de las diferentes isoenzimas del citocromo P- 450

- No se recomienda el uso de secuestradores de sales biliares, ácido nicotínico ni inhibidores de la absorción de colesterol (ezetimiba)

- Para el tratamiento de la hipertrigliceridemia se recomienda el uso de gemfibrozil, ciprofibrato o fenofibrato

- La lovastatina y la simvastativa no deben combinarse con la TARGA

- El inicio de la terapia farmacológica se hará tomando en cuenta los lineamientos del NCEP III

- Una vez iniciado el tratamiento con estatinas o fibratos deben monitorizarse a los 0, 3, 6 meses y luego cada 6 meses los niveles séricos de transaminasas

- El monitoreo rutinario de los niveles de creatinfostoquinasa ( CPK ) no esta recomendado
- El uso concomitante de fibratos y estatinas debe evitarse

- La respuesta al tratamiento farmacológico se evaluará a las 6 semanas de su inicio

\begin{abstract}
Lipid disorders are one of the most frequent complications of HIV positive patients receiving antiretroviral treatment. The intervention of these patients is difficult, especially due to the interactions among some lipid lowering drugs and protease inhibitors. The guides presented here are facilitate the diagnosis, classification an aimed to intervention of these patients.
\end{abstract}

\section{Referencias}

1. Dronda F. Riesgo vascular en pacientes con infección crónica por el VIH -1:controversias con implicaciones terapéuticas, clínicas y pronósticas. Enferm Infecc Microbiol Clin 2004;22:40-5

2. Henry K, Melroe H, Huebsch J et al. Severe premature coronary artery disease with protease inhibitors ( letter ). Lancet 1998;351:1328

3. Bozzette SA, Ake CF, Tam HK et al. Cardiovascular and cerebrovascular events in patients treated for human immunodeficiency virus infection. N Engl J Med 2003;348:702-10

4. Grinspoon S, Carr A. Cardiovascular risk and body - fat abnormalities in HIV - infected adults. N Engl J Med 2005;352:48-62

5. Mauss S, Stechel J, Willers R et al. Differentiating hyperlipidaemia associated with antiretroviral therapy. AIDS 2003;17:189-94

6. Gallant JE, Staszewski AL, Pozniak E et al. Efficacy and safety of Tenofovir DF vs Stavudine in combination therapy in antiretroviralnaïve patients : a 3 - year randomized trial. JAMA 2004;292:191-201

7. Yeni PG, Hammer MS, Hirsch MS et al. Treatment for adult HIV infection : 2004 recommendations of the International AIDS Society - USA Panel. JAMA 2004;292:251-65

8. Dubé MP, Stein JH, Aberg JA et al. Guidelines for the Evaluation and Management of Dyslipidemia in Human Immunodeficiency Virus (HIV)-Infected Adults Receiving Antiretroviral Therapy: Recommendations of the HIV Medicine Association of the Infectious Disease Society of America and the Adult AIDS Clinical Trials Group. Clin Infect Dis 2003;37:613-27

9. Expert Panel on Detection Evaluation and Treatment of High Blood Cholesterol in Adults. Executive summary of the third report of the National Cholesterol Education Program (NCEP) Expert Panel on Detection, Evaluation, and Treatment of High Blood Cholesterol in Adults (Adult Treatment Panel III). JAMA 2001; 285:2486-97

10. Grundy SM, Cleeman JI, Bairey Merz N et al. Implications of recent clinical trials for the National Cholestrol Education Program Adult Treatment Panel III Guidelines. Circulation 2004;110:227-39 